\title{
Influência do exercício concorrente sobre as variáveis metabólicas de idosas fisicamente ativas em condição alimentada
}

\author{
Influence of concurrent exercise on metabolic variables in physically active \\ elderly women fed condition
}

\author{
Marcelo Costa Junior ${ }^{1}$ \\ Leandro Pereira de Moura' \\ Natalia Oliveira Bertolini' ${ }^{1}$ \\ Inaian Pignatti Teixeira ${ }^{1}$ \\ Maria Alice Rostom De Mello' \\ Eduardo Kokobun ${ }^{1}$ \\ Eliete Luciano ${ }^{1}$ \\ 1. Laboratório de Nutrição \\ Metabolismo e Exercício/Fisiologia \\ Experimental, Núcleo de Atividade \\ Física Esporte e Saúde, Instituto de \\ Biociência, Unesp Rio Claro-SP
}

\begin{abstract}
Resumo
O aumento exacerbado do número de morbidade e mortalidade advindas das doenças crônico-degenerativas gera um aumento do gasto publico destinados a saúde. A concentração plasmática de triglicerídeos pós-estado alimentado aponta-se como um fator preditor para a formação do processo aterosclerótico com possível progressão para doenças cardiovasculares. Em contrapartida, o exercício físico apresenta-se como importante estratégia para redução dos níveis de triglicerídeos circulantes, por promover aumento da atividade da enzima lipase lipoproteica. $\mathrm{O}$ objetivo do presente estudo foi analisar o efeito do exercício físico combinado de intensidade leve em variáveis metabólicas de idosas fisicamente ativas em situação pós-prandial. Foram selecionadas para esse estudo 20 idosas (média de 68,02 \pm 9,06 anos participantes a mínimo um ano do Programa Saúde Ativa Rio Claro, com frequência de duas vezes por semana. $O$ estudo foi dividido em dois dias sendo que no primeiro dia foram coletadas medidas antropométricas, inquérito alimentar e nível de atividade habitual. O segundo dia foi composto por um protocolo de atividade física de intensidade leve dividida em três etapas; alongamento, exercício aeróbio e exercício resistido. Foram realizadas coletas sanguíneas nos momentos pré e pós exercício. Foi observada redução significativa na glicemia em função do exercício físico combinado, porém não houve diferença na concentração de triglicerídeos nos momentos pré e pós. Conclui-se que exercício físico concorrente de caráter leve pode promover redução da glicemia e manutenção dos triglicerídeos em idosas fisicamente ativas na condição alimentada.
\end{abstract}

\section{ENDEREÇOPARACORRESPONDÊNCIA}

Marcelo Costa Júnior Departamento de Educação Física Universidade Estadual Paulista (UNESP) Avenida 24a 1515 - Caixa Postal 199 Bela Vista, Rio Claro, SP, Brasil 13506-900 marcelotmcosta@hotmail.com

$\begin{array}{ll}\text { • Recebido: } & 05 / 09 / 2011 \\ \text { • Re-submissão: } & 08 / 11 / 2011 \\ & 27 / 11 / 2011 \\ \text { • Aceito: } & 29 / 11 / 2011\end{array}$

\begin{abstract}
The exacerbated increase of morbidity and mortality due chronic diseases leads to an increase public expenditures for health. The plasma triglyceride post-fed state has been mentioned as a predictor for the formation of the atherosclerotic process with possible progression to cardiovascular disease. In contrast, exercise has become an important strategy for reducing levels of circulating triglycerides by promoting increase of lipoprotein lipase activity. Analyzes the effects of light combined physical exercise in metabolic variables in elderly physically active post-prandial women. Twenty elderly women (mean $68.02 \pm 9.06$ years) who had attend of Active Health Programme / Rio Claro-SP, Brazil per one year, often twice a week. The study was divided into two days and in the first day were collected anthropometric measurements, inquiry dietary and level of habitual activity. The second day consisted of a protocol of light physical activity divided into three parts, stretching, aerobic exercise and resistance exercise. Blood samples were collected before and after workout. A significant reduction in blood glucose due to the combined exercise was observed, but no difference in triglycerides concentration before and after workout was found. We conclude that light combined physical exercise can promote reduced serum glucose and maintain the triglycerides concentration in physically active elderly in the fed condition.
\end{abstract}




\section{INTRODUÇÃO}

Nos últimos anos houve um aumento exacerbado do número de morbidade e mortalidade decorrentes das doenças cardiovasculares (DCV $)^{1}$, bem como de doenças crônico-degenerativas ${ }^{2}$, ocasionando em um aumento do gasto público destinado a tais patologias ${ }^{3}$.

Dentre os vários fatores que podem influenciar no aparecimento ou em maiores complicações da DCV podemos destacar a formação do processo aterosclerótico que é considerado um dos principais preditores desta condição ${ }^{4}$. A aterosclerose e consequente disfunção endotelial são fenômenos pós-prandiais que podem estar relacionados com a lentificação da remoção das lipoproteínas ricas em triglicérides (TG) da circulação sanguínea ${ }^{5}$. Assim, a concentração plasmática de triglicérides no estado pós alimentado (TGpp) e não a de jejum, aponta-se como um fator preditor mais fidedigno na patogênese e progressão das doenças cardiovasculares ${ }^{6}$, mesmo comparado com fatores de risco tradicionalmente aceitos ${ }^{7}$.

Outro fator importante a ser considerado é a glicemia, visto que uma condição hiperglicêmica influencia o risco de lesão das artérias contribuindo para a disfunção endotelial e consequente formação da placa ateromática ${ }^{8}$. O diabetes mellitus e as dislipidemias, patologias comuns na população idosa brasileira, tem como característica a elevação da glicemia e TG circulantes.

Em contrapartida, o exercício físico apresenta-se como importante estratégia para redução dos níveis de triglicerídeos circulantes, por promover aumento da atividade da enzima lipase lipoproteica (LPL) ${ }^{9}$ e também reduzir a glicemia devido a uma melhor captação de glicose ${ }^{10}$, assim retardando e/ou prevenindo a formação de placa aterosclerótica. Contudo, estudos nessa linha encontram resultados contraditórios. $\mathrm{Na}$ literatura, existem evidências de redução transitória nas concentrações de TGpp ${ }^{11,12}$ em consequência do exercício físico, quanto da manutenção ou elevação das concentrações de TGpp ${ }^{13,14}$.

As diferenças podem ser decorrentes dos diferentes ergômetros usados nos protocolos de exercício físico: esteira"11, cicloergômetro ${ }^{14}$ e resistido $9,15,16$, ou das diferentes intensidades (de moderada a altas intensidades) e volumes (30 min a $120 \mathrm{~min}$ ) empregadas nos protocolos. Existem poucos estudos que utilizam exercícios resistidos e aeróbios combinados.

O horário e a quantidade calórica da ingestão são situações que devem ser levadas em consideração. Vários estudos verificaram o efeito do exercício em relação a refeições igualitárias ao almoço e ao mesmo horário dessa refeição ${ }^{9,11,15,16}$, contudo é de interesse verificar outros horários e ingestões menos calóricas como após o café da manha.

Visando à redução da alta taxa de sedentarismo brasileira, nos últimos anos foram criados diversos programas com o objetivo de incentivar a prática de atividade física e melhorar a Qualidade de Vida da população ${ }^{17}$. Há 10 anos foi criado, no município de Rio Claro/SP - Brasil, o Programa Saúde Ativa Rio Claro ${ }^{18}$ que oferece atividades físicas as 15 unidades básica de saúde da cidade. As sessões de atividade física ocorrem duas vezes por semana com uma hora de duração e são compostas por exercícios tanto aeróbios como resistidos de intensidade leve.

Devido ao considerável aumento na prevalência de sedentarismo, diabetes mellitus, e dislipidemia na população moderna, falta consenso na literatura sobre este assunto. Estudos envolvendo a concentração de TGpp e glicemia em resposta a exercício físico de leve intensidade em idosas tornam-se necessários que avaliem tais situações. Portanto o objetivo do presente estudo foi analisar o efeito do exercício físico combinado de intensidade leve em variáveis metabólicas em idosas fisicamente ativas em situação pós-prandial.

\section{MÉTODOS}

\section{Voluntários}

Após aprovação do Comitê de Ética em Pesquisa da Universidade Estadual Paulista - UNESP - Rio Claro-SP (protocolo 0847), participaram deste estudo 20 mulheres com média de $68,02 \pm 9,06$ anos e que estivessem participando a no mínimo um ano do Programa Saúde Ativa Rio Claro, com frequência de duas vezes por semana.

\section{Delineamento Experimental}

Na primeira etapa do estudo foram coletadas medidas antropométricas, inquérito alimentar e o nível de atividade física avaliado pelo questionário $\mathrm{IPAQ}^{19}$. Foram incluídas na amostra apenas as idosas consideradas ativas (com mais de 150 min de atividade física semanal).

$O$ inquérito alimentar teve o intuito de diagnosticar a alimentação das voluntárias durante o café da manha, e para que os sujeitos não modificassem seu hábito alimentar foram incluídas somente as idosas que ingeriam uma xicara de café (com uma colher de açúcar) e um pão francês (com margarina) o que corresponde a aproximadamente 320 calorias.

Após uma semana, no mesmo período do dia, foi realizado o protocolo de exercício físico de leve intensidade com coletas de amostras sanguíneas nos momentos pré e pós exercício físico. Foi recomendado aos avaliados que a refeição do café da manhã fosse mantida e que evitassem exercício físico extenuante nas 24 horas que antecedesse o protocolo de exercício.

\section{Protocolo de exercício agudo}

O exercício físico foi realizado após uma hora da ingestão do café da manhã. A sessão de exercício físico foi dividida em duas fases:

1. Fase inicial: Esta fase foi constituída de um alongamento inicial durante 5 minutos com ênfase na parte inferior do corpo. Após o alongamento as idosas caminharam por 5 minutos, sendo que, durante a caminhada elas realizaram quatro atividades:
a. Andar na ponta dos pés;
b. Andar com o calcanhar;
c. Andar de costas;
d. Andar lateralmente.

2. Fase principal: Durante a fase principal foram dados estímulo de aumento da capacidade aeróbia e de resistência muscular, unindo assim os benefícios gerados pelo exercício aeróbio e resistidos. Essas atividades foram subdivididas em três períodos:

a. Caminhada em intensidade leve por $15 \mathrm{~min}$ em um percurso plano de aproximadamente 800 metros;

b. Atividade priorizando a parte lúdica estimulando o componente aeróbio por cerca de 10min: brincadeira "coelhinho sai da toca". Nesta atividade foram feitos círculos no chão em ambos os lados da quadra, ao sinal da frase "coelhinho sai da toca" os participantes ocuparam o outro circulo feito do outro lado da quadra, ou seja, quem estava de um lado deveria procurar uma "toca" do outro lado e vice -versa, quem ficassem sem o circulo dá o sinal para próxima troca.

c. Caminhada de 20 minutos, com paradas para a realiza- 
ção de exercício. Esses exercícios foram divididos em duas etapas com 10 minutos cada uma:

i. Os participantes caminhavam pela quadra segurando um bastão, ao sinal do instrutor as voluntarias paravam e realizavam exercícios de força, depois voltavam a caminhar livremente pela quadra. Foram realizadas 20 repetições para cada exercício que englobavam as musculaturas do quadríceps, adutores, abdutores, panturrilha e abdômen.

ii. "Step adaptado" - Era feito um círculo no chão para cada participante. Em seguida as mesmas posicionavam atrás do círculo e realizavam deslocamentos básicos (Para frente, lados e para trás) de forma que realizassem uma cruz, mantendo como referência o círculo feito no chão.

\section{Medidas Antropométricas}

A massa corporal foi verificada em balança antropométrica com precisão de $100 \mathrm{~g}$ (Plenna) e a estatura em estadiômetro de madeira com escala de $0,1 \mathrm{~cm}$. A partir das medidas de massa corporal e estatura, calculou-se o índice de massa corporal (IMC) em $\mathrm{Kg} / \mathrm{m}^{2}$. A circunferência de cintura foi medida em duplicata, no ponto entre a última costela e a crista ilíaca, utilizando-se uma fita métrica flexível com precisão em milímetros. As medidas antropométricas foram realizadas em todos os momentos pelo mesmo avaliador.

\section{Dosagens Bioquímicas Plasmáticas}

Nos momentos pré e pós exercício físico foram coletados $25 \mu$ l de sangue da polpa digital, para dosagens bioquímicas plasmáticas de triglicérides e glicemia pelo método espectrometria através de kit comercial Laborlab ${ }^{\circledR}$.

\section{Análise Estatística}

A normalidade dos dados foi verificada através do teste de Shapiro Wilk, sendo apresentadas como média \pm desvio padrão. A comparação entre os momentos pré e pós-exercício físico foram realizadas através do teste t-Student para amostras dependentes. Como critério para diferença significativa foi adotado $p<0,05$. Todas as análises estatísticas foram realizadas através do software Statistica 7.0 - Statsoft.

\section{RESULTADOS}

A Tabela 1 apresenta dados antropométricos das voluntárias. Nessa tabela pode-se observar um Índice de Massa Corporal considerada grau I de obesidade e uma Razão Cintura Quadril enquadradas como risco moderado.

Foi observada redução significativa na glicemia em função do exercício físico combinado (Fig. 1). Entretanto, não houve diferença entre os momentos pré e pós nas concentrações de triglicerídeos, porém o exercício físico pode ter evitado esse aumento promovendo uma manutenção dessas concentrações (Fig. 2).

\section{DISCUSSÃO}

Diante dos resultados encontrados, verifica-se a importância da prática regular de exercícios físicos combinados de intensidade leve, com o propósito de se obter um menor efeito deletério à saúde no estado pós-alimentado reduzindo significativamente a glicemia e evitando um possível aumento das concentrações de triglicérides.

O pico glicêmico pós-prandial depende da quantidade de carboidratos, tipo e composição da refeição e, ainda, do horário do dia em que a refeição é realizada. Sabe-se, por exemplo, que após o café da manhã o pico é maior do que em outros momentos do dia ${ }^{20}$. Considerando que o nível da glicemia começa a aumentar dez minutos após a ingestão de alimentos e atinge os seus valores máximos aos 60 minutos após ingestão alimentar, a atividade física pode ser uma ferramenta eficiente na amenização do pico da glicemia e retorno da mesma aos seus valores normais.

Alguns estudos ${ }^{21}$ demonstram que uma única sessão de exercício físico aumenta a captação de glicose mediada pela

\begin{tabular}{cccccc} 
MC (kg) & EST (m) & $\mathbf{C A}(\mathbf{c m})$ & $\mathbf{C Q}$ (cm) & RCQ & IMC \\
\hline $67,51 \pm 9,80$ & $1,55 \pm 0,59$ & $89,66 \pm 13,18$ & $102,37 \pm 8,68$ & $0,87 \pm 0.99$ & $27,99 \pm 4,39$ \\
\hline
\end{tabular}

Nota: RCQ (Razão Cintura Quadril); IMC (Índice Massa Corporal ); MC (Massa Corporal); EST (Estatura); CA (Circunferência Abdominal) e CQ (Circunferência Quadril).

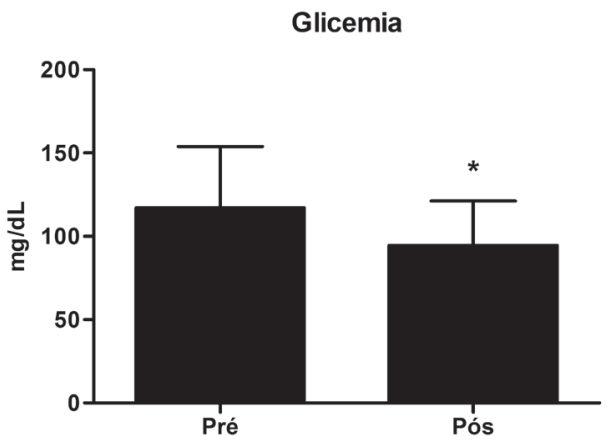

Figura 1
Concentração de glicose sanguínea nos momentos pré e pós exercício. *diferença significativa com $\mathrm{p}<0,05$
Triglicérides

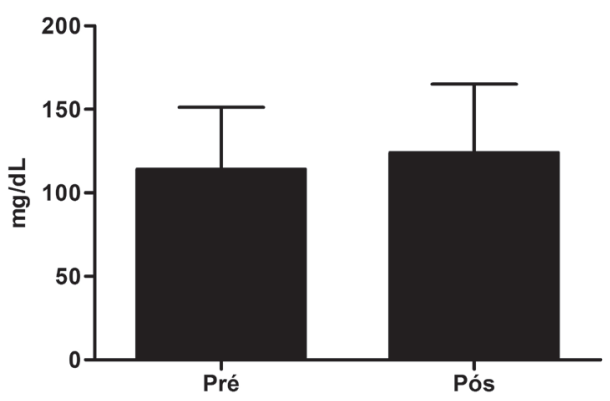

Figura 2 Concentração de triglicérides sanguíneo nos momentos pré e pós exercício 
insulina tanto em sujeitos normais, como em sujeitos resistentes à insulina, obesos com resistência à insulina, e diabéticos do tipo 2, e o exercício físico crônico melhora a sensibilidade à insulina em indivíduos saudáveis, em obesos não-diabéticos e em diabéticos dos tipos 1 e 2.

Larsen e colaboradores ${ }^{22}$ observaram decréscimos consideráveis na glicemia e nas concentrações de insulina durante o exercício e também no período pós-exercício, quando comparados ao controle. O estado pós exercício pode ser caracterizado por um período em que a capacidade de captação da glicose melhora devido a dois fenômenos: $O$ efeito residual da captação de glicose estimulada pela contração muscular, que é independente de insulina e o aumento da sensibilidade à insulina ${ }^{23}$.

A sensibilidade à insulina aumentada no período pós exercício está relacionada com a translocação dos transportadores de glicose muscular GLUT4 ${ }^{24}$. No entanto fatores, além daqueles envolvendo translocação do GLUT4 também podem contribuir para o fenômeno. Segundo Maarbjerg e colaboradores ${ }^{25}$, a ação de insulina está restrita aos músculos ativos e causada, principalmente, por aumento da translocação de GLUT4 para a membrana da célula muscular. Além disso, a permeabilidade da membrana, acompanhada pela elevação da insulina estimula a perfusão microvascular no estado pós-exercício que poderia favorecer a captação de glicose adicional.

Assim, talvez o efeito da contração muscular combinado de várias pequenas ampliações na sinalização insulínica e na perfusão microvascular podem explicar a grande melhoria em relação à ação da insulina sobre a captação de glicose após uma única sessão de exercício.

No que se refere ao efeito agudo do exercício na concentração de triglicérides, são escassos os estudos que verificaram a combinação do exercício resistido e aeróbio de intensidade leve. No presente estudo verificou-se que a combinação dessas diferentes modalidades de exercício físico leve promoveu a manutenção das concentrações de triglicérides em idosas fisicamente ativas.

Segundo Teixeira e colaboradores ${ }^{26}$, uma refeição pode modificar o padrão normal da curva de lipemia pós-prandial, aumentando os níveis plasmáticos de triglicérides mesmo quando associada com exercício. Bloomer e colaboradores ${ }^{27}$, mostraram que homens ou mulheres que praticavam exercícios de caráter anaeróbio ou aeróbio respectivamente e isoladamente não tiveram alteração na resposta pós-prandial em relação a indivíduos sedentários e também não mostraram alteração entre os protocolos de treinamento. Tal afirmação evidencia a importância do protocolo empregado nesse estudo, por evitar os aumentos das concentrações de triglicérides podendo contribuir para prevenção da aterosclerose e consequente desenvolvimento de doenças cardiovasculares correlatas.

Corroborando com esses achados Belli e coloboradores ${ }^{28}$ verificaram redução significante dos triglicerídeos pós-prandiais em função do exercício físico agudo de intensidade progressiva (teste de vai-e-vem de $20 \mathrm{~m}$ ) em indivíduos jovens fisicamente ativos, evidenciado esses efeitos preventivos. Diante desses achados torna-se evidente o efeito agudo do exercício físico na redução ou manutenção na concentração triglicérides, porém ainda não está bem esclarecido quais os mecanismos primordialmente envolvidos nesse processo fisiológico.

Entretanto, existem algumas hipóteses para tentar explicar estes aspectos. Segundo Spriet ${ }^{29}$, indivíduos treinados têm uma maior capacidade de estocar triglicérides nos mús- culos podendo aumentar a capacidade de oxidação e utilização para o fornecimento de energia, além disso, possuem um maior número de mitocôndrias e níveis aumentados de enzimas envolvidas na síntese aeróbia de ATP, facilitando a utilização dos triglicérides durante a atividade física ${ }^{30}$. Outro benefício evidente promovido pelo treinamento físico é o aumento da concentração da enzima Lipase Hormônio Sensível (responsável pela degradação dos triglicérides), e aumento da atividade da enzima lipase lipoproteica $(L P L)^{9}$ promovendo assim, um incremento do consumo e fornecimento de energia para a contração muscular proveniente dos lipídeos. A amostra desse estudo foi constituída de idosas fisicamente ativas, sendo que a manutenção na concentração de triglicérides possivelmente ocorreu devido a tais fatores advindos do beneficio promovido pelo treinamento físico.

Cabe ressaltar que outros estudos relataram reduções similares em exercícios de moderada intensidade ${ }^{11,12}$, enquanto outros evidenciaram a intensidade moderada como sendo a mais efetiva para promover tal resposta ${ }^{11,15}$. O contraste entre os resultados de outros trabalhos quando comparados ao nosso, possivelmente deve-se ao tempo de coleta pós-exercício ou a intensidade de exercício. No presente estudo, as coletas foram realizadas imediatamente após o exercício físico, enquanto Belli e colaboradores ${ }^{28}$, analisaram os TGpp 4h após o exercício físico em contraste com estudos prévios ${ }^{11-13}$ que utilizaram modelo de 2 dias de intervalo (exercício físico no $1^{\circ}$ dia e teste pós-prandial no $2^{\circ} \mathrm{dia}$ ).

Nossos achados sugerem que o exercício combinado agudo em mulheres idosas pode ser uma importante estratégia terapêutica não medicamentosa para o controle da dislipidemia e hiperglicemia no estado alimentado, porém mais estudos deverão ser realizados para reforçar estas evidências. O achado mais importante do presente estudo esta relacionado à intensidade e utilização do protocolo concorrente, nessa situação onde uma sessão aguda de exercício físico de intensidade leve realizado após uma pequena refeição, respectiva ao café da manhã, reduz a glicemia e promove manutenção dos valores dos triglicerídeos séricos. Essa intensidade utilizada foi de importante valia visto que idosos não suportam e/ ou sentem um desconforto muito grande com intensidades moderadas e altas.

\section{CONCLUSÃO}

Portanto podemos concluir que exercício físico concorrente de caráter leve pode promover redução da glicemia e manutenção dos triglicerídeos em idosas fisicamente ativas na condição alimentada. Importante ainda ressaltar que o protocolo utilizado neste estudo é interessante para tal população, tendo em vista que idosas podem ter várias outras limitações que impeçam a realização de exercícios de moderada e alta intensidade.

\section{REFERENCIAS}

1. ORGANIZAÇÃO MUNDIAL DA SAÚDE. Hipertension: Facts. 2010 [acessado 01 de novembro de 2010]; Disponível em: http://www.who.int/dietphysicalactivity/publications/facts/diabetes/en/>.

2. Silva CA, Lima WC. Efeito benéficos do exercício físico no controle metabólico do diabetes mellitus tipo 2 à curto prazo. Arquivos Brasileiros de Endocrinologia e Metabologia 2002;46(5):550-6.

3. Roberts CK, Barnard J. Effects of exercise and diet on chronic disease. J Appl Physiol 2005;98:3-30.

4. Gomes FPA, Carmo TGM. Dislipidemia pós-prandial e doença cardiovascular Rev Bras Nutr Clin 2006;21(1):60-71

5. Zilversmitt DB. Atherogenesis: a postprandial phenomenon. Circulation 1979:60:473-485.

6. Patsch W, Esterbauer $\mathrm{H}$, Foger B, et al. Postprandial lipemia and coronary risk. Curr Atheroscler Rep 2000;2:232-242. 
7. Roche HM, Gibney MJ. Effect of long-chain polyunsaturated fatty acids on fasting and postprandial triacylglycerol metabolism. Am J Clin Nutr 2000;71:S232-S7.

8. Cruzes LA, Bosco TEC, Pandini VE, et al. Hiperglicemia Pós-Prandial em Pacientes com Diabetes Melito Tipo 2. Arq Bras Endrocrinol Metab 2008;52(4):642-8.

9. Burns SF, Broom DR, Miyashita M, et al. Increased Postprandial Triacylglycerol Concentrations following resistance exercise. Med Sci Sports Exerc 2006;38(3):527-33.

10. American Diabetes Association. Standards of medical care in diabetes-2006. Diabetes Care 2006;29(1):S4-S42.

11. Tsetsonis NV, Hardman AE. Effects of low and moderate intensity treadmill walking on postprandial lipaemia in healthy young adults. Eur J Appl Physiol 1996;73:419-26.

12. Zhang JQ, Ji LL, Fretwell VS, et al. Effect of exercise on postprandial lipemia in men with hypertriglyceridemia. Eur J Appl Physiol 2006;98:575-82.

13. Pfeiffer $M$, Wenk $C$, Colombani PC. The influence of 30 minutes of light to mod erate intensity cycling on postprandial lipemia. European Journal of Cardiovascular Prevention and Rehabilitation 2006;13:363-8

14. Cambri LT e Santos RL. Influência dos exercícios resistidos com pesos em diabéticos tipo 2. Motriz 2006;12(1):33-41.

15. Magkos F, Tsekouras $\mathrm{Y}$, Prentzas $\mathrm{K}$, et al. Acute exercise-induced changes in basal VLDL-triglyceride kinetics leading to hypotriglyceridemia manifest more readily after resistance than endurance exercise. J Appl Physiol 2008;105(4):1228-36

16. Hallal PC, Tenório MCM, Tassitano RM, et al. Avaliação do programa de promoção da atividade física Academia da Cidade de Recife, Pernambuco Brasil: percepções de usuários e não-usuários. Cad. Saúde Pública [online] 2010;26(1):70-78

17. Nakamura PM, Chiyoda A, Gomes GAO, et al. Programa De Intervenção Para $A$ Prática De Atividade Física: Saúde Ativa Rio Claro. Revista Brasileira de Atividade Física e Saúde 2010;15:128-32.

18. IPAQ - International Physical Activity Questionnaire. Guidelines for data processing and analysis of the International Physical Activity Questionnaire (IPAQ) - short and long forms. 2005. [acessado 29 de dezembro de 2010]; Disponível em: <http://www.ipaq.ki.se/dloads/IPAQ\%20LS\%20Scoring\%20Protocols Nov05.pdf>.

19. Tuomilehto J. A glucose tolerance test is important for clinical practice. Diabetes Care 2002;25:1880-2.

20. Kirwan JP, Kohrt WM, Wojta DM, et al. Endurance exercise training reduce glucose-stimulated insulin levels in 60- to 70 -year-old men and women. J Gerontol 1993;48:M84-90.

21. Miller JP, Pratley RE, Goldberg AP, et al. Strength training increases insulin action in healthy 50- to 65-yr-old men. J Appl Physiol 1994;77:1122-7

22. Larsen JJ, Dela F, Madsbad S, et al. The effects of intense exercise on postprandial glucose homeostasis in type II diabetic patients. Diabetologia 1999:42(11):1282-92.

23. Richter EA, Garetto LP, Goodman MN, et al. Enhanced muscle glucose me tabolism after exercise: modulation by local factors. Am J Physiol 1984;246: E476-82.

24. Geiger $\mathrm{PC}$, Han $\mathrm{DH}$, Wright $\mathrm{DC}$, et al. How muscle insulin sensitivity is regulated: testing of a hypothesis. Am J Physiol Endocrinol Metab 2006;291:E1258-63.

25. Stine JM, Lykke S, e Erik AR. Current understanding of increased insulin sensitivity after exercise - emerging candidates. Acta Physiologica 2011;202(3):32335

26. Teixeira M, Kasinski N, Izar MCO, et al. Efeitos do exercício agudo na lipemia pós-prandial em homens sedentários. Arquivos Brasileiros de Cardiologia 2006;87(1):3-11

27. Bloomer RJ; Ferebee $\mathrm{DE}$, Fisher-Wellman $\mathrm{KH}$, et al. Postprandial Oxidative Stress: Influence of Sex and Exercise Training Status. Medicine \& Science in Sports \& Exercise 2009;41(12):2111-19.

28. Belli T, Coelho FGM, Ferreira SA, et al. Impacto do exercício físico agudo no perfil metabólico pós prandial em adultos fisicamente aptos. Rev Bras Cineantropom Desempenho Hum 2009,11(3):314-9.

29. Spriet LL. Regulation of skeletal muscle fat oxidation during exercise in humans. Med Sci Sports Exerc 2002;34:1477-84.

30. Maglischo EW. Nadando ainda mais rápido. $1^{\text {a }}$ ed., São Paulo, Editora Manole, 1999. 